\title{
Internacionalización de
}

\section{la economía colombiana.}

Las políticas de

apertura económica,

modernización industrial

y tecnológica

ARCADIO JOSE GUZMAN NOGALES

Profesor Universidad del Valle

\section{Introducción}

Ste artículo presenta los rasgos
fundamentales de la Politica de
Internacionalización de la

Economía Colombiana, con énfasis en las políticas de Comercio Exterior, Modernización Industrial y

Tecnológica.

Inicialmente se hace un diagnóstico y se describen los objetivos generales de la Política de Apertura Económica, sus instrumentos y estrategias. Más adelante se analizan las politicas sectoriales mencionadas anteriormente. Por último, y ya que se trata de un proceso reciente, se presentan algunas conclusiones provisionales acerca del impacto real de la política global sobre el proceso de desarrollo industrial.
I. Diagnóstico y objetivos generales de la política de apertura económica.

a política se fundamenta en un
diagnóstico que critica el modelo
de desarrollo "hacia adentro", tradicionalmente seguido en el país (una combinación de sustitución de importaciones - protección a la industria nacional y promoción de exportaciones); señalando que "la protección de los mercados internos frente a la competencia externa ha generado distorsiones en los precios relativos y ha conducido a que la producción nacional se aísle de la competencia internacional, limitando los avances en términos de crecimiento, acceso a tecnología moderna, productividad, exportaciones, empleo y satisfac- 
ción de las necesidades de los consumidores a precios razonables." Se complementa el diagnóstico planteando que la protección a la industria nacional ha creado un "sesgo antiexportador" en la economía colombiana al reducir la rentabilidad de los sectores potencialmente exportadores y propiciar inversiones (con base en rentabilidad artificial) en actividades que no son las de mayor potencialidad de crecimiento ni las que mayores posibilidades tienen de orientarse al mercado externo. Estas actividades, al ser más intensivas en la utilización de capital que de trabajo, desaprovechan la amplia disponibilidad de mano de obra que tiene el país.

Basado en este diagnóstico el gobierno colombiano establece los grandes objetivos de su política:

"Superar los obstáculos estructurales al crecimiento económico y crear las condiciones requeridas para mejorar las condiciones (sic) de empleo e ingreso, y elevar los niveles de bienestar de la población".

"Adelantar un proceso de modernización de la economía..., y dinamización del aparato productivo".

"Avanzar en un proceso de internacionalización de la economía..., y modernización del aparato productivo".

En síntesis se critica el modelo proteccionista de industrialización por sustitución de importaciones basado en el mercado interno y se propone uno de restructuración industrial y desarrollo

(1) Programa de Modernización de la Economia Colombiana. Documento CONPES DNP-2465-J Revista de Planeación y Desarrollo. Volumen XXII, Números I y 2 -enero-junio 1990. Departamento Nacional de Planeación. Bogotá, Colombia. económico basado en la competencia y el mercado externos. ${ }^{i z}$

\section{Instrumentos y estrategias de la política de apertura económica.}

Para el logro de dichos objetivos y en desarrollo del "PROGRAMA DE MODERNIZACION DE LA ECONOMIA COLOMBIANA" ${ }^{\eta_{33}}$, el gobierno nacional ha venido adoptando una serie de medidas en las áreas de:

a) Comercio exterior y política cambiaria

b) Modernización industrial

c) Ciencia y tecnología

d) Sector financiero

e) Infraestructura y transporte

f) Reforma laboral

g) Sector agrario

h) Inversión extranjera

Para los fines del presente artículo el análisis se concentrará en las medidas tomadas en comercio exterior y política cambiaria, modernización industrial y política de ciencia y tecnología. Estas áreas están teórica y prácticamente relacionadas. A continuación se presenta un análisis sintético del proceso adelantado en cada una de ellas.

\section{A. Comercio Exterior y Política Cambiaria.}

Según el gobierno, la política de racionalización del comercio exterior debe ser entendida como la "continuación y pro-
(2) Ibid.
(3) Ibid. 


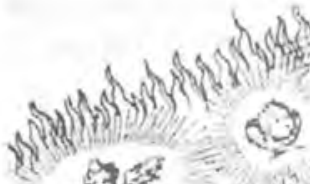

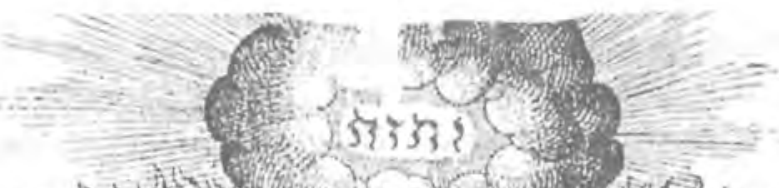

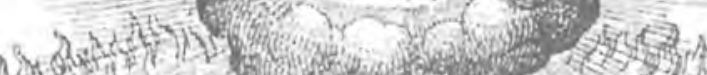

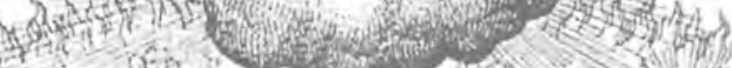

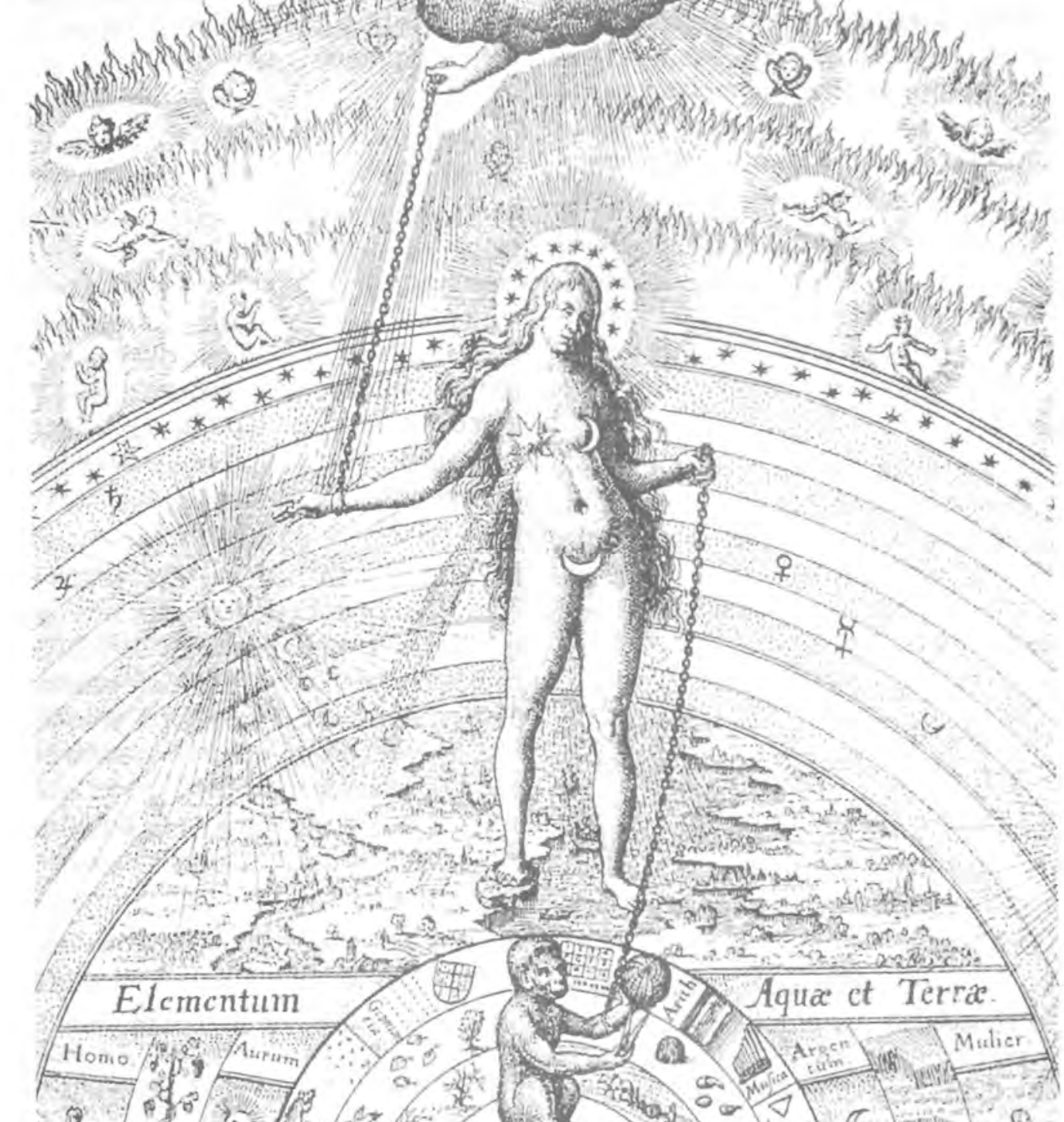

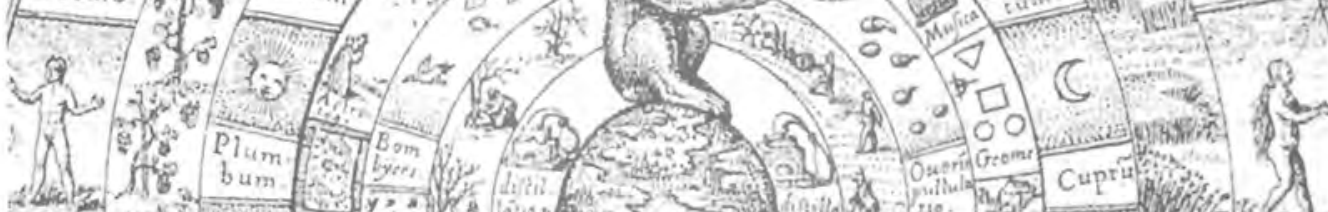

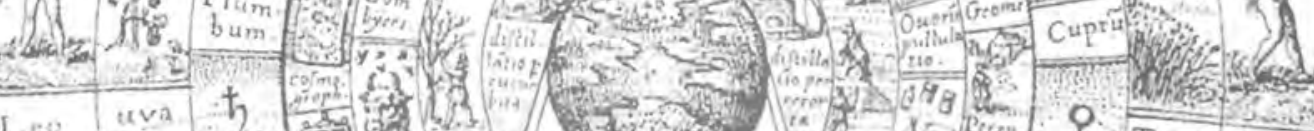

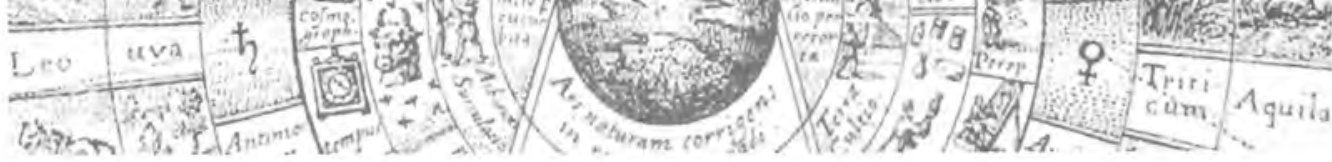


fundización del proceso de modernización de la economía colombiana", con esta política se busca introducir una competencia gradual pero progresiva a la producción nacional y, simultáneamente, continuar la reducción de los controles administrativos sobre las importaciones de bienes complementarios con dicha producción. ${ }^{(4)}$ Las medidas tomadas están basadas en cuatro criterios fundamentales de carácter general:

1. El de automaticidad, mediante el cual se buscó minimizar el grado de discrecionalidad en el manejo de las importaciones (en la práctica su aplicación llevó a la eliminación del régimen de licencia previa).

2. El de universalidad, tratando de evitar discriminaciones en favor o en contra de sectores específicos y buscando que toda la producción nacional quede expuesta a la competencia externa, si bien en un grado controlado.

3. El de gradualidad, según el cual la política está lejos de constituir una política de choque o una apertura abrupta de la economía y por lo tanto debe permitir un proceso de adecuación en el tiempo de la estructura productiva del país a las condiciones de una mayor apertura de la economía al resto del mundo.

4. El de sostenibilidad, según el cual la sostenibilidad del proceso no sólo está garantizada por su gradualidad sino por su consistencia con la política macroeconómica y con las perspectivas de la balanza de pagos del país.

Según el gobierno, en una primera etapa de dos años, se iniciaría la exposición de la producción nacional a la competencia externa, mediante una reducción progresiva de las restricciones cuantitativas a las importaciones sustituyendo mediante protección arancelaria la protección que era otorgada a través del mecanismo de la licencia previa.

Una vez centrado el control de las importaciones en el arancel y la tasa de cambio, en una segunda etapa de tres años, se profundizaría el grado de competencia externa a través de una reducción gradual y sostenida del arancel y de su grado de dispersión, hasta alcanzar un nivel cercano al $25 \%$, incluyendo la sobretasa arancelaria, meta considerada como el nivel de protección deseable en una perspectiva de mediano plazo y justificable en condiciones normales de productividad.

El propósito básico de esta primera etapa sería entonces lograr un proceso de transición hacia condiciones de mayor competencia y eficiencia, sin generar desequilibrios en la actividad productiva, y profundizar en él en la medida en que lo permitieran las condiciones macroeconómicas, cuidándose de observar rigurosamente los criterios mencionados anteriormente. ${ }^{(5)}$

En la práctica la política de comercio exterior ha sido ejecutada a tal velocidad que crea serias dudas acerca de su gradualidad: en un período de siete meses se invirtió casi totalmente el régimen de importaciones; antes de febrero 22/90 el $38.9 \%$ de las posiciones arancelarias (1999) eran de libre importación, el $60.1 \%$ (3.090 posiciones) estaban en régimen de licencia previa y el $1.0 \%$ (54 
posiciones) eran de prohibida importación. El proceso de liberación de importaciones fue tan veloz que en septiembre $18 / 90$ ya se había llegado a la siguiente situación: el $77.0 \%$ de las posiciones arancelarias (3.940) estaban en régimen de libre importación, el $23.0 \%$ (1.218 posiciones) estaban en régimen de licencia previa y había quedado abolido el régimen de prohibida importación. ${ }^{(6)}$

Simultánea y paralelamente a este proceso de liberación de importaciones el gobierno flexibilizó el régimen de licencia previa hasta eliminarlo definitivamente. En virtud de dicho proceso de flexibilización se realizaron las "encuestas arancelarias" con el fin de asignar cupos de divisas para importaciones con base en el sobre arancel ofrecido; la característica predominante de los ciclos de encuestas fue, además de los bajos gravámenes adicionales, la poca demanda para la importación de los bienes encuestados, de tal forma que el cupo de divisas establecido para cada grupo no se utilizó en su totalidad para estas primeras licencias de importación adjudicadas.

Adicionalmente el gobierno modificó el arancel de aduanas, reduciendo los impuestos a las importaciones y la dispersión arancelaria existente, lo que le implicó un sacrificio fiscal significativo, ${ }^{(7)}$

En este marco se plantearon serios interrogantes acerca del impacto de la política sobre la estructura de la distribución del ingreso, pues el gobierno redujo el impuesto CIF a las importaciones del $18 \%$ al $13 \%$, buscando contribuir a la

(6) La Apertura Económica en Colombia: Agenda de un proceso. Cámara de Comercio de Bogotá. Oficina de Planeación y Desarrollo. Bogotá: La Cámara 1990.

(7) Ibid. mayor competitividad en los costos de la producción nacional abaratando la importación de maquinaria, insumos y bienes de capital y trató de compensar el efecto fiscal desfavorable elevando el IVA del 10 al 12\%. Paralelamente el gobierno dispuso una mayor tasa de devaluación fijando un tope del $30 \%$ al concluir 1990; es bien sabido el efecto inflacionario de la devaluación que se constituye así en otro factor que empeora la distribución del ingreso.

Complementariamente se ha tomado toda una serie de medidas tendientes a consolidar una infraestructura de apoyo administrativo al comercio exterior: la Junta Monetaria autorizó al Banco de la República a elevar en US\$100 millones la disponibilidad de divisas de los establecimientos financieros para facilitar las operaciones en moneda extranjera de las empresas que necesitan comprar materias primas u otros bienes en el exterior y eliminó las garantías para el reintegro de las divisas de las exportaciones dando a los exportadores amplios plazos para reintegrar el producto de sus ventas sin las garantías y controles previos a que los sometía el Estatuto Cambiario.(8)

Por su parte la Dirección General de Aduanas, autorizó a todos los bancos comerciales del país para que recibieran el pago de los derechos de importación, impuestos y demás gravámenes y multas que afecten a las mercancías que se despachen por las aduanas, rompiendo así el monopolio administrativo que en este aspecto poseía el Banco de la República. ${ }^{(9)}$

(8) Tbid.

(9) Ibid 
Para evitar que la flexibilización del manejo de las importaciones facilite el contrabando, la subfacturación y el dumping se planteó el establecimiento de otras medidas adicionales de control y soporte administrativo: la creación de un sistema de información de los precios de las importaciones en el mercado internacional; la creación de un registro de importadores; el fortalecimiento técnico y operativo de la aduana y la adopción de un estatuto antidumping. Las acciones anteriores fueron complementadas con otras medidas de apoyo, como son: la descentralización del Plan Vallejo, la agilización de sus trámites, la eliminación del registro previo de exportación y la reestructuración del Incomex y Proexpo para dar paso a la creación del Ministerio de Comercio Exterior y el Banco de Comercio Exterior. ${ }^{\text {wo }}$

\section{B. Modernización Industrial}

Para "apoyar los cambios que el proceso conlleva y lograr al mismo tiempo las metas de incremento de exportaciones", el Gobierno, "contando con el apoyo técnico y financiero de los organismos internacionales" (Banco Mundial), afirmó que pondría en marcha un programa de reestructuración y reconversión de la base productiva, con base en estudios realizados para cinco subsectores considerados representativos: automotor, cuero y calzado, textiles, hierro y acero, y alimentos."in

Estos estudios (que efectivamente fueron realizados) tenían como propósito fundamental, "la identificación de elemen-

(10) Ibid.

(II) Programa de Modernización de la Economia Colombiana. tos y procedimientos" que habrían de caracterizar el programa, con la finalidad de que a él tuvieran acceso todas las unidades productivas que queriendo llevar a cabo modificaciones en su estructura de producción, presentaran a consideración de las entidades pertinentes, programas debidamente sustentados en términos de su viabilidad económica y de sus requerimientos.

Se hacía notar desde un principio, que para la reestructuración y reconversión era "conveniente" el apoyo gubernamental y que por lo tanto el gobierno pondría a "disposición de los empresarios que operan en el país instrumentos financieros, técnicos y de desarrollo social que les ayuden a reestructurar sus operaciones, estimulando la adquisición, adaptación y generación de tecnología nueva".

La promoción al desarrollo tecnológico se complementaría con tres acciones fundamentales:

1. La modernización de maquinaria y equipo, mediante la nueva política arancelaria y el sistema de crédito industrial.

2. La modificación de los criterios utilizados por el Comité de Regalías, para dar mayor efectividad a la reglamentación sobre selección y transferencia de tecnología.

3. El fortalecimiento institucional de la investigación tecnológica.

Adicionalmente, se intensificarían y reformularían programas de asistencia al proceso de adecuación de la fuerza laboral a las nuevas condiciones, orientados a acelerar su reentrenamiento, mejorar su capacitación y facilitar la movilidad vertical y horizontal en el mercado de 
trabajo.

Ya que en la práctica la ejecución de la política industrial es responsabilidad básica del Ministerio de Desarrollo y del Instituto de Fomento Industrial, parece razonable tratar de deducir los principales lineamientos de la ejecución de la política de la forma como ésta es conceptualizada por ambas instancias.

Para el Ministerio de Desarrollo, la política económica no se puede reducir a la simple liberación de importaciones ya que lo que se pretende es sentar unas nuevas bases sobre las cuales se pueda afirmar el proceso de desarrollo social y económico en lo que resta del presente siglo logrando la re-inserción de la economía colombiana en el escenario internacional. Lo anterior implica un replanteamiento a fondo de la política industrial que le permita al sector readquirir el papel dinamizador del progreso económico. $^{.12)}$

En esta conceptualización, la gradualidad es criterio indispensable para el proceso de desgravación arancelaria de los próximos cuatro años. El proceso comenzará a partir de 1991, lo que significa que los empresarios tendrán un año inicial para ajustarse al esquema y tres años más para mejorar sus niveles internacionales de competencia. Al final del proceso el arancel máximo será del 23\% (uno de los más altos de América Latina), y habrá cuatro niveles arancelarios (protección discriminada por tipos de bienes). Las primeras reducciones serán para los bienes de capital no producidos en el país y

(12) Bases de la Nueva Politica Industrial en Medio de la Apertura. Palabras del Ministro de Desarrollo Económico, Ernesto Samper Pizano. Clausura de la 46a. Asamblea Anual de Afiliados de la ANDI. Bucaramanga, Noviembre 16, 1990. materias primas destinadas a la producción nacional, habrá diferentes velocidades en el programa de desgravación de tal manera que los empresarios tendrán un horizonte claro de liberación arancelaria al cual podrán referir sus decisiones inmediatas y futuras de ajuste y reconversión.

En este esquema la licencia previa sólo será un instrumento de protección excepcional (en caso de desequilibrios internacionales) y la protección a la industria nacional estará dada básicamente por el arancel, el estatuto antidumping y la política de tasa real de cambio, complementados con controles administrativos para evitar entrada masiva de saldos y bienes usados ("mercancía chatarra").

Para el Ministerio de Desarrollo no todos los subsectores industriales están gradualmente preparados para acometer el reto de la internacionalización, en consecuencia adopta el criterio de selectividad en la ejecución de la política industrial para no someter a una injusta desprotección a sectores o industrias que debido a las condiciones de la competencia internacional necesitan que su apertura funcione a ritmo diferente. En consecuencia el Ministerio identifica cinco subsectores industriales:

1. Industrias tradicionales que por la naturaleza de los productos que generan y los altos costos y dificultades para su transporte internacional, deberán orientarse prioritariamente al mercado nacional: alimentos, bebidas y muebles de madera y metal.

2. Industrias tradicionales que ya han alcanzado aceptables posibilidades de competencia externa y que requieren un apoyo definido del gobierno en calidad, 
especialización, canales de comercialización y abastecimiento de materias primas: acero, calzado, textiles, confecciones, imprentas y algunos bienes agroindustriales.

3. Industrias con claro perfil exportador y que requieren un fuerte apoyo tecnológico y en algunos casos, aportes considerables de nueva inversión, especialmente extranjera: química industrial, petroquímica, plásticos y caucho.

4. Industrias que pueden resultar seriamente afectadas por la apertura y que requieren programas inmediatos de reconversión y especialización que, acompañados de un plan más lento de desgravación arancelaria, les permitan ajustarse al compromiso de la internacionalización: siderúrgica, autopartes, automotriz y fertilizantes.

5. Industrias incipientes o inexistentes de alto potencial exportador hacia las cuales se dirigirán los esfuerzos de promoción de inversión de la nueva política industrial: bienes de capital, sectores electrónicos y bienes asociados al empleo intensivo de la biotecnología.

Partiendo de esta base la política industrial se plantea como objetivos: hacer competitiva a la industria nacional en términos de precios internacionales, desarrollar programas que la acerquen a la meta de la calidad total, especializarse en ramas, relocalizar los nuevos desarrollos industriales en las costas y controlar la acción de formas monopólicas. ${ }^{(13)}$

Para el logro de lo anterior la Junta Monetaria estudia nuevas condiciones crediticias para estimular la relocaliza-

(13) Ibid. ción de los nuevos proyectos económicos en las costas y el IFI y PROEXPO tratarán de desconcentrar geográficamente una cartera que en la actualidad está aplicada en más de un $48 \%$ y $83 \%$, respectivamente en las tres principales ciudades del país.

El desarrollo exportador se conseguiría con el rediseño de los instrumentos tradicionales de promoción de exportaciones (CERT, Plan Vallejo, Zonas Francas) y su articulación al nuevo Ministerio de Comercio Exterior.

La apertura, por sí sola, no traerá el aumento del crecimiento si no están dadas unas condiciones de productividad y eficiencia. Para ésto el gobierno debe inducir, a través de una mayor inversión, el facilitamiento de la adquisición de tecnología internacional y el desarrollo de una política de ciencia y tecnología que asigne el mejoramiento de las condiciones de producción.

Según el Ministerio de Desarrollo los enunciados anteriores constituyen las bases de la nueva política industrial. ${ }^{(14)}$

El IFI por su parte propone un "Plan Nacional de Reconversión Industrial"(15) según el cual las actividades económicas se clasifican en tres categorías:

1) Las que serán sometidas a "estrés"

2) las de poca "intensidad tecnológica" y 3) las actividades estratégicas.

La primera de estas categorías incluye aquellas actividades sometidas a un proceso de presión competitiva a causa de la

(14) Ibid.

(15) Criterios para la Reconversión Industrial, Marco Legal y el papel del IFI. Ministerio de Desarrollo Económico, Instituto de Fomento Industrial. Gerencia. Bogotá, Colombia. Noviembre 20 de 1990. 


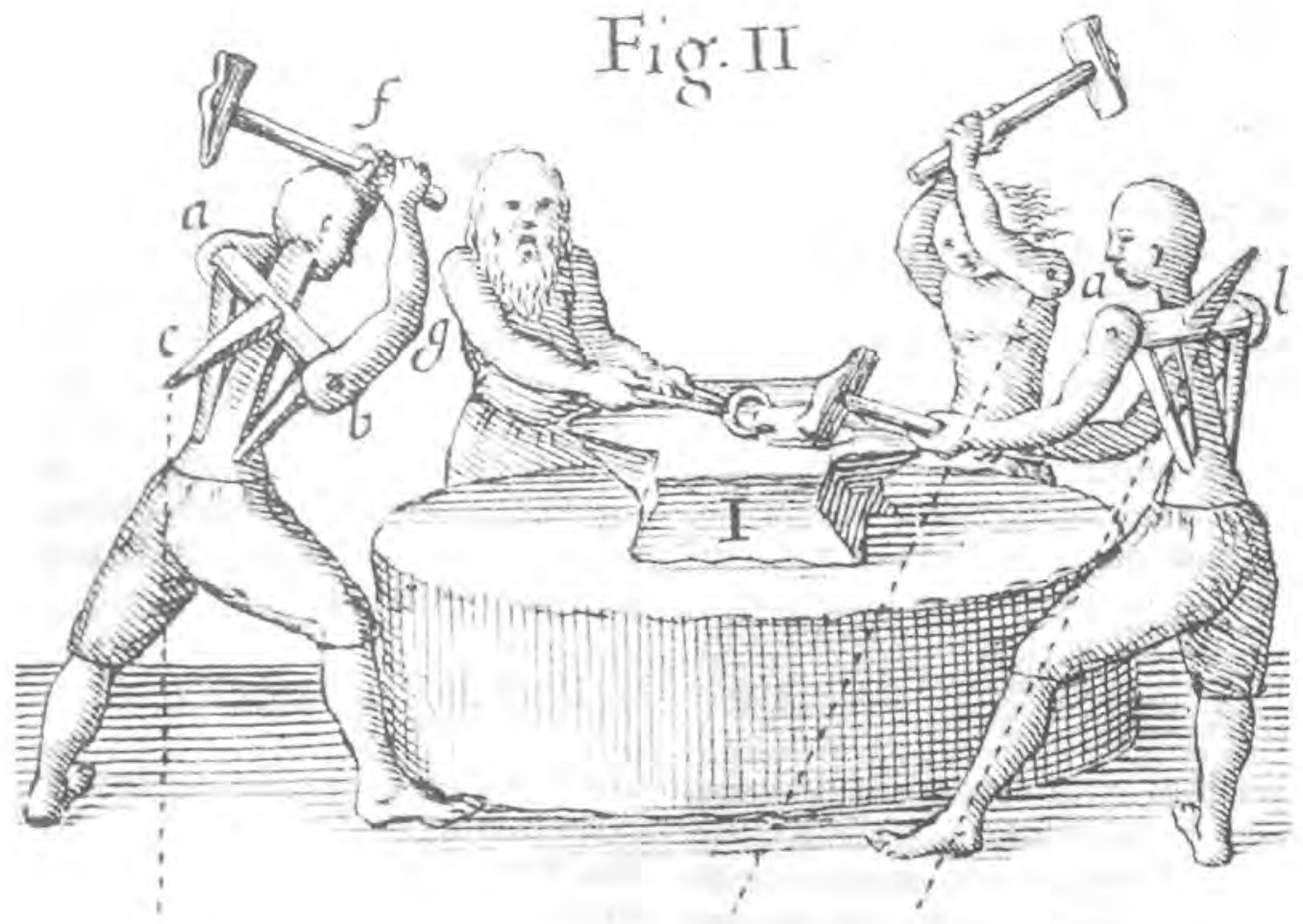

desgravación arancelaria, la que tendrá el efecto de disminuir la rentabilidad de la producción para los mercados internos.

En consecuencia, aquellos sectores (Siderúrgico, metalmecánico, químico y petroquímico) cuya protección efectiva disminuya en mayor medida se verán forzados a elevar su nivel de eficiencia para poder sobrevivir, a la vez que algunos de éstos verán contraídos sus niveles de actividad.

Las actividades de poca intensidad tecnológica son aquellas que en el corto plazo más rápidamente pueden contribuir a elevar el empleo y atraer divisas.

Las actividades estratégicas son las que cumplen con los objetivos del desarrollo nacional en razón de las siguientes características: generan una proporción relativamente alta de valor agregado nacional; tienen efectos dinámicos en el resto de la economía; ofrecen posibilidades de cambio tecnológico para aumentar la productividad y constituyen sectores dinámicos del comercio internacional.

La política de reconversión industrial sería aplicable a las actividades de poca "intensidad tecnológica" y las que se consideran estratégicas. El criterio básico que se utilizaría para seleccionar tales actividades es que, al promover su desarrollo, sean capaces de impulsar el crecimiento del desarrollo industrial y de la economía en general. Como criterios adicionales se tendrían en cuenta el 
tamaño actual de la actividad y el ritmo al cual crece o puede crecer, el grado de progreso tecnológico y la productividad laboral. Adicionalmente se establecería el criterio de "eficiencia en la estructura productiva", analizando las actividades en "vía de reconversión" de acuerdo con indicadores como la participación del grupo industrial en la generación de exportaciones del sector manufacturero y la generación de exportaciones a terceros mercados, complementando estos criterios con indicadores como el valor de la materia prima nacional empleada por el grupo industrial, el grado de integración, las ventas de la actividad a sectores productivos nacionales y su peso relativo dentro del valor agregado total del sector industrial, lo mismo que el salario promedio por trabajador. ${ }^{(16)}$

Con base en la experiencia de países como España, Italia y México, el IFI elaboró el borrador de un proyecto de ley "por el cual se establece un esquema de incentivos con el fin de promover la reconversión del sector industrial colombiano y estimular su competitividad internacional". El proyecto establece que el Ministerio de Desarrollo y el Departamento Nacional de Planeación podrán declarar en "reconversión" sectores de la industria y la agroindustria y grupos de empresas en particular. Esta declaratoria se producirá cuando se "considere que la competitividad económica del respectivo sector o grupo de empresas tiene importancia estratégica para el comercio internacional del país". (17)

Formulada la iniciativa por parte del Ministerio de Desarrollo o de Planea-

(16) Ibid.

(17) Ibid. ción Nacional, y con carácter previo a la declaración, el Consejo Nacional de Política Económica y Social, Conpes, solicitará al IFI la elaboración del correspondiente proyecto de Programa de Reconversión Industrial que deberá contener la descripción comparada de la situación nacional e internacional del sector y el programa de inversiones prioritarias para asegurar su competitividad internacional. Las empresas de los sectores sometidos a reconversión deberán a su vez elaborar un programa interno de reconversión, cuyo seguimiento y control estaría a cargo del IFI (convertido en Corporación Nacional para el Desarrollo a partir de 1991).

\section{Política Tecnológica}

Con la política de apertura, modernización e internacionalización de la economía, el componente tecnológico pasa a ser un elemento fundamental de toda la política de desarrollo. Partiendo del diagnóstico de un efecto negativo del modelo proteccionista sobre el desarrollo tecnológico de la industria (la industria aislada de la competencia externa desarrolló un "sesgo anti-exportador", los avances conseguidos en la productividad durante estas décadas se consiguieron más por incrementos de capital o de mano de obra que por nuevas combinaciones de estos factores resultantes de la asimilación de innovaciones tecnológicas) se propone ahora hacer del componente tecnológico la base de la productividad industrial.

(18) Tecnología para el Proceso de Apertura. Palabras del Ministro de Desarrollo Económico. Acto de Instalación del Primer Congreso de ACOPLASTICOS, Bogotá, D.E.. octubre 22. 1990. 
El objetivo inicial de la nueva política tecnológica será atenuar el efecto recesivo producido por la apertura, en su primera etapa, que afecta principalmente a las industrias menos avanzadas y que requieren la pronta implantación de nuevos procesos administrativos, técnicos y de distribución, es decir, de innovación tecnológica. ${ }^{199}$

En búsqueda de los mencionados objetivos la política tecnológica se mueve en tres direcciones fundamentales:

1. La reorganización institucional del sistema nacional de ciencia y tecnología.

2. La búsqueda de nuevas fuentes de financiamiento y apoyo para la producción de tecnología.

3. La concertación con el sector empresarial en la búsqueda de un nuevo concepto de calidad.

\section{a) La reorganización institucional.}

En desarrollo de lo establecido por la ley 29/90 por medio de la cual se planteó la reorganización del sistema de ciencia y tecnología, el Departamento Nacional de Planeación, el Ministerio de Desarrollo Económico y el Ministerio de Educación, elaboraron una propuesta de reorganización institucional que, a partir de COLCIENCIAS como eje articulador de toda la política, permita organizar la producción, asimilación y difusión del conocimiento técnico y científico. ${ }^{120 !}$

En lo pertinente a tecnología extranjera, se reconoce que Colombia seguirá siendo, por muchos años, una importadora neta de tecnología, por lo tanto el gobierno tratará de facilitar las condi-

(19) Ibid.

(20) Ibid. ciones para que la importación de tecnología se haga en condiciones de selectividad y oportunidad. Selectividad para que las técnicas importadas correspondan realmente a las necesidades de innovación en el sector productivo y oportunidad para que trámites excesivos no impidan la rápida canalización de esta tecnología. Se avanza en la aprobación automática de regalías y se trabaja en una propuesta para acelerar el registro de nuevas marcas, adicionalmente y para evitar la especulación con marcas extranjeras se exigirán certificacicnes que acrediten la autenticidad de la propiedad de dichas marcas. ${ }^{21}$

En lo que a tecnología nacional se refiere, el gobierno cree que la responsabilidad de la creación tecnológica no es exclusiva del Estado. En principio, el gobierno asumirá la producción de tecnología en los sectores de salud, educación, vivienda, así como en los bienes básicos para el consumo o estratégicos para el desarrollo. La tecnología privada será responsabilidad del sector empresarial en la medida en que sea evidente el carácter de apropiabilidad de los beneficios que ella genera. A manera de ejemplos exitosos de investigación científica privada se mencionan los centros experimentales como CENICAFE, CENICAÑA y el INSTITUTO DEL PETROLEO que son considerados como importantes avances en el camino de construir un nuevo perfil tecnológico sectorial. Para estimular la innovación privada se plantea la creación de un sistema de estímulos tributarios, financieros y cambiarios y una acción concertada del gobierno y el sector privado en

(21) Ibid. 
el diseño de "incubadoras industriales a través de las cuales el Estado y un sector empresarial puedan financiar el desarrollo de proyectos de investigación tecnológica". (22)

Tan importante como la producción de tecnología es su asimilación. Para desarrollar la capacidad del país, de asimilación tecnológica el Ministerio de Desarrollo está trabajando en el diseño de un Banco de Tecnología como instrumento de ejecución de la Política de Asimilación tecnológica, el cual conectado con sistemas internacionales de información tecnológica deberá sistematizar todos los registros tecnológicos, nacionales y extranjeros, organizar el sistema de propiedad intelectual, servir de enlace entre los productores nacionales y los mercados mundiales de tecnología y mantener una permanente divulgación sobre nuevas técnicas y procesos que puedan ser aprovechados por los empresarios para mejorar sus condiciones de competencia. En este proceso de masificar la apropiación de la tecnología, aparece la necesidad de extender procesos de "integración industrial" a través de los cuales las industrias más modernas se encadenen a las más pequeñas y medianas para hacerlas partícipes de la modernización. El proceso de asimilación debe llegar a definir las bases de un criterio de "Desagregación tecnológica" que supere a la figura de los contratos "llave en mano" que eliminan cualquier posibilidad de vinculación de la industria nacional a proyectos de complejo contenido tecnológico. ${ }^{137}$

Complemento indispensable de la gene-

(22) Ibid.

(23) Ibid. ración y asimilación de la tecnología es su difusión. Para conseguirla, la nueva política trabajará conjuntamente con el SENA, el ICFES y el SISTEMA UNIVERSITARIO en el diseño e implementación de nuevos canales de difusión tecnológica, partiendo del supuesto de que en ocasiones es más difícil distribuir que generar tecnología. La propuesta incluye el desarrollo de un programa de becarios en el exterior, de alta calificación y el cambio en las normas de inmigración para permitir el ingreso al país de personal altamente calificado.

Para financiar la política se cuenta con dos líneas específicas: una para COLCIENCIAS por valor de US\$64 millones y otra de fondos japoneses por US\$150 millones. La pequeña y mediana empresa, por su parte, cuenta con un apoyo específico del BID para asistencia técnica cuya utilización está siendo reprogramada por la Corporación Financiera Popular. Adicionalmente, está la línea de reconversión industrial acordada con el Banco Mundial que es, en esencia, una línea de financiación tecnológica en la medida en que dirigirá sus fondos hacia el mejoramiento de las condiciones administrativas, tecnológicas y de distribución de la industria colombiana. En forma complementaria el gobierno reactivará una línea de PROEXPO dirigida a modernización productiva y existe la determinación de canalizar nueva inversión extranjera hacia el país como una manera de disminuir la dependencia del crédito externo y obtener la financiación de nuevos frentes de producción y de trabajo por medio de inversión extranjera que asuma riesgo aportando capital y tecnología. ${ }^{(24)}$

(24) Ibid. 
El programa también tendrá el apoyo del fondo de modernización de la economía previsto en la nueva Ley Marco de Comercio Exterior. A este Fondo, para financiamiento tecnológico, se llevarán los impuestos generados por la compra y transferencia de tecnologías, impuestos que suman aproximadamente US\$35 millones anuales.

La política tecnológica tiene como norte la meta de la calidad. El Ministerio de Desarrollo conjuntamente con el sector empresarial, dará impulso a los centros de calidad. De la misma manera, está preparando un programa cuatrienal que tiene como propósito abrir camino al concepto de calidad total.

La calidad que se piensa exigir a los productores nacionales, para competir en el exterior, también les será exigida a los competidores externos en Colombia. Para evitar la inundación de "mercancías chatarra", es decir, mercancías de calidad inferior, saldos de segunda, bienes reconstruidos y sustancias tóxicas cuya distribución ha sido prohibida en los países que las exportan, el Ministerio de Desarrollo aplicará las normas pertinentes contenidas en el Decreto 2746 de 1984, exigiendo certificación de calidad para los productos importados. ${ }^{1251}$

\section{Conclusiones provisionales, dado el carácter incipiente del proceso}

Del análisis realizado se pueden deducir los grandes lineamientos de la política económica del presente gobierno y señalar sus aspectos más relevantes para el desarrollo futuro de la industria colombiana.

(25) Ibid.
La política económica gubernamental de modernización e internacionalización de la economía colombiana comprende un conjunto muy amplio de medidas destinadas a superar el agotamiento del modelo de sustitución de importaciones que, según el diagnóstico, ha sido causa del lento crecimiento económico de los últimos años. Para los propósitos de este artículo, el análisis se restringió a los campos de Comercio Exterior, política industrial y política tecnológica.

La política económica, evidentemente, ha concentrado el mayor número de medidas en el campo del Comercio Exterior. Ya desde fines del gobierno anterior se había tomado la decisión de liberar importaciones, pero este proceso se aceleró fuertemente en el gobierno actual hasta llegar a una situación en la cual el 96.8\% del arancel quedó bajo el régimen de libre importación y fue eliminado el régimen de licencia previa, que había sido el criterio básico de protección a la industria nacional en el anterior modelo de sustitución de importaciones. La reforma al comercio exterior, sin embargo, va más allá de la liberación de importaciones y se inscribe dentro del concepto más general de "internacionalización" consagrado en la Ley Marco de Comercio Exterior (Ley 1 de 1991) que establece como criterios generales de la política de comercio exterior: el fomento del comercio libre de bienes y servicios, la libertad de impulso a la modernización y la eficiencia, y el apoyo a los procesos de integración internacional. La ley establece que los instrumentos de política de comercio exterior podrán reglamentarse de acuerdo con la situación externa del país en cada momento, con sujeción de los anteriores criterios. 
Esto implica que las restricciones al comercio (tales como los controles cuantitativos, etc.) podrán ser sólo temporales pero cambia además el criterio con que se pueden imponer estos controles, pues ya no será el de la protección a la industria nacional, sino la defensa de las reservas internacionales en caso de crisis externa..$^{26}$

En materia de aranceles, la reforma establece la necesidad de su reducción y homogeneización. Para ello el gobierno ha anunciado un cronograma de desgravación arancelaria que va hasta 1991 con el propósito, en el corto plazo, de lanzar señales claras para evitar el comportamiento especulativo de los importadores a la espera de nuevas reducciones y, más a largo plazo, para facilitar el proceso de reestructuración industrial.

La reforma al sector externo incluye también un componente de reforma ins-

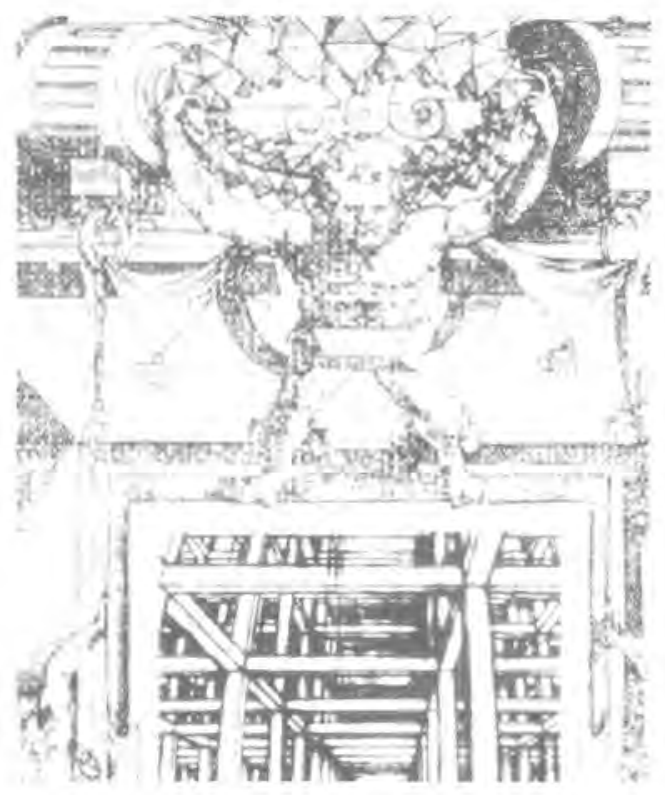

(26) Reformas para la modernización de la economía colombiana. FEDESARROLLO. Bogotả, D.E. Revista Coyuntura Económica. Abril 1991. titucional con el fin de adaptar los organismos existentes - dedicados en gran parte en gran parte al manejo de los controles cuantitativos - a los nuevos criterios generales de la política. Para ello el nuevo Ministerio de Comercio Exterior centralizará todas las labores relacionadas con esta actividad, las cuales estaban anteriormente a cargo del Ministerio de Desarrollo y de otros ministerios. Se crea también el Banco del Comercio Exterior que asumirá su papel de promotor del sector exportador como banco internacional y un Fondo de Modernización Económica para financiar proyectos de infraestructura relacionados con el sector externo y, en general, proyectos que promuevan las exportaciones. Se estableció también la creación del Consejo Superior de Comercio Exterior, que será el encargado de definir las políticas de exportación, importación, aranceles y, en general, de la reglamentación de los instrumentos correspondientes.

En cuanto a la Reforma Cambiaria, la Ley 9 de 1991 otorga al gobierno amplias facultades para regular el mercado cambiario. Mantiene los instrumentos de control previamente existentes pero le permite manejarlos en forma totalmente discrecional. Según la Ley 9/91 los ingresos provenientes de servicios prestados al exterior por residentes en el país no tienen la obligación de negociarse en el Banco de la República o en los intermediarios autorizados, lo cual legaliza de hecho un mercado paralelo de divisas y podría sentar las bases para la "dolarización de la economía". El gobierno ha manifestado que mantendrá el sistema de mini-devaluaciones, pero que habrá una tendencia a que la tasa de 
cambio sea más flexible en la medida en que el mercado libre empiece a cobrar importancia.

\section{Algunos interrogantes a} manera de conclusiones

Del análisis realizado se puede deducir el trazo grueso inicial de la política económica del presente gobierno, señalar sus puntos más relevantes y plantear algunos interrogantes.

Tal y como está planteada por parte del Gobierno Nacional, la política de apertura no parece ser una expresión de aperturismo externo, como el que inspira al Banco Mundial y en general a los sectores radicales del neoliberalismo económico.

Un perfil más moderado que expresaria criterios de desarrollo industrial, parece configurarse en el discurso y las declaraciones oficiales del Ministerio de Desarrollo. Así parecen indicarlo no sólo algunos objetivos explícitos (mejorar empleo e ingreso, modernizar y dinamizar el aparato productivo) sino también los criterios de gradualidad y selectividad mantenidos en el proceso de desgravación arancelaria (plazo de tres años y definición de cuatro niveles arancelarios); los deseos de fundamentar la política de Modernización Industrial en estudios sectoriales serios (automotriz, cuero y calzado, textiles, hierro y acero, alimentos); los cambios institucionales realizados tendientes a consolidar una infraestructura administrativa de apoyo al proceso; la definición de una política tecnológica y el reconocimiento de su importancia estratégica en el proceso de desarrollo así como el propósito de concertación con el sector empresarial en la búsqueda de un nuevo concepto de calidad.

En sentido contrario a esta tendencia de desarrollo industrial, opera la política macroeconómica de corto plazo que lidera el Ministerio de Hacienda con una directriz fundamental antinflacionaria claramente definida. La política macroeconómica de corto plazo conspira contra el crecimiento de la producción y el empleo productivos y debilita la tendencia "Industrializante" del Ministerio de Desarrollo: la política monetaria contraccionista del crédito eleva los niveles de las tasas reales y nominales de interés esterilizando en la práctica la operatividad del crédito disponible para modernización y reestructuración industrial. La política de devaluación encarece los equipos importados necesarios para la modernización, agudiza el servicio de la deuda externa tanto para el sector público como para el privado, acelera la inflación y empeora la distribución del ingreso.

Si tenemos en cuenta además el desfase temporal que media entre las políticas monetaria, cambiaria y de comercio exterior, cuyos efectos son casi inmediatos, y las políticas industrial y tecnológica cuyos resultados son de mediano y largo plazos, no parece descabellado suponer que la industria colombiana ya esté siendo sometida a un proceso de reestructuración regido por la competencia y el mercado externos, que puede conducir en el futuro a una "primarización" económica en concordancia con las ventajas comparativas históricamente establecidas en nuestro país y en detrimento de una estrategia de desarrollo autónomo con mayor empleo y bienestar para los colombianos. 


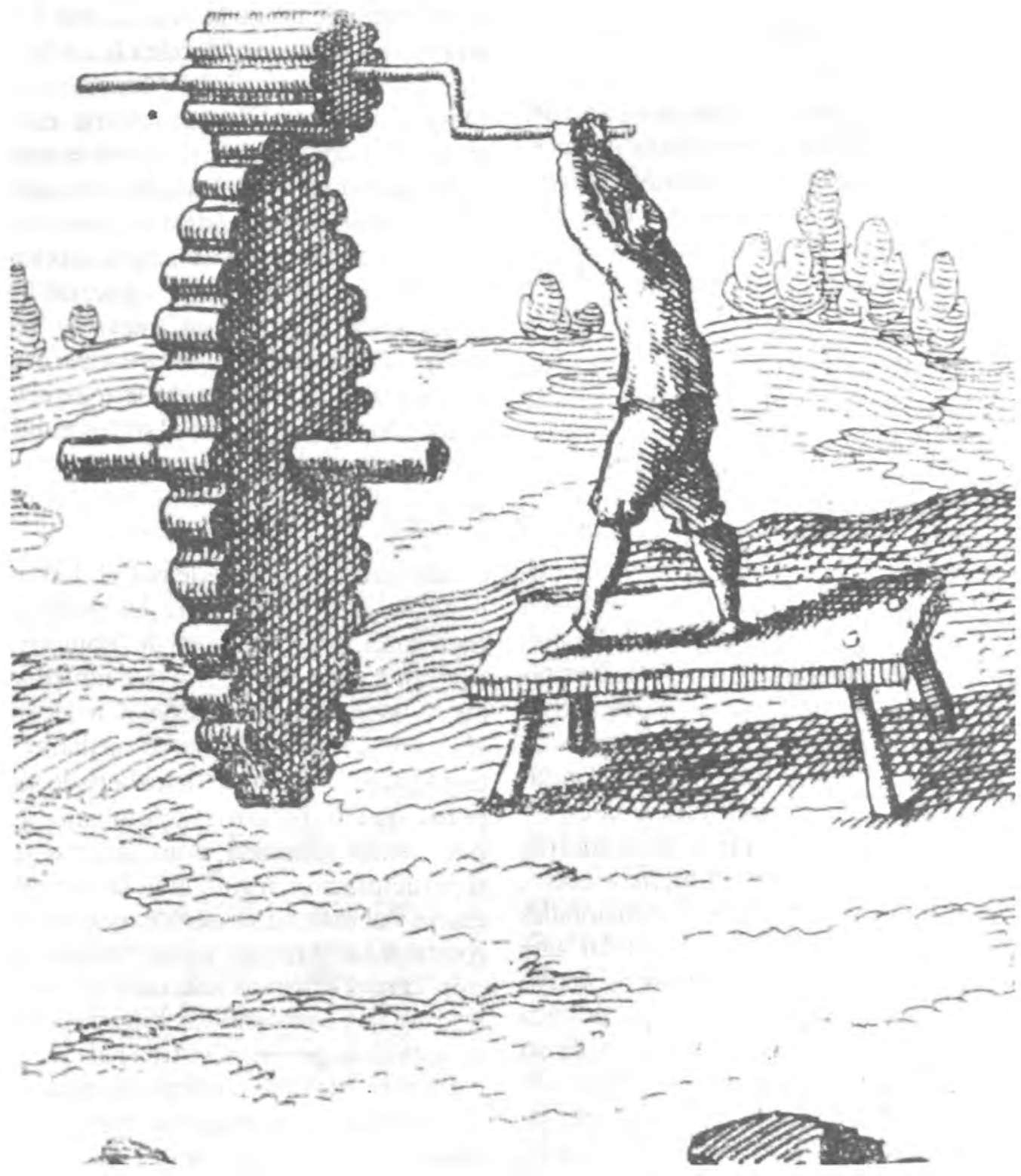




\section{Bibliografias}

\section{Algunos elementos geopolíticos del Pacto Cafetero}

ARANGO, Mariano. El Café en Colombia. Carlos Valencia Editores, Bogotá, 1982.

NIETO Arteta, Luis Eduardo. El Café en la sociedad colombiana, coedición ECOE, Bogotá, 1975.

OCAMPO, José Antonio -editor. Lecturas de economía cafetera. Tercer mundo y Fedesarrollo, Bogotá, 1987.

PALACIOS, Marco. El Café en Colombia 1950-1970. El áncora editores, México, 1983.

RAMIREZ Ocampo, Jorge y PEREZ, Silverio, 83 años de política cafetera internacional y la participación de Colombia en este proceso. Federación, Bogotá, 1984.

REVEIZ, Edgar -Compilador-, La cuestión cafetera. Colección debates. CEDE, Bogotá, 1980.

FEDERACION BOGOTA. Propósitos de la industria cafetera 1850-1986. Junio 1987.

FEDESARROLLO. Debates de coyuntura económica No. 15 - El rompimiento del Pacto Cafetero, su impacto económico. Bogotá, 1989.

FEDESARROLLO. Coyuntura Económica Volumen XX No. 1. Marzo, 1990.

FEDESARROLLO Y FONDO CULTURAL CAFETERO. Economía Cafetera Colombiana. Bogotá 1980.

FEDERACION NACIONAL DE CAFETEROS. Ensayos sobre Economía Cafetera No. 4. Abril-septiembre, 1989.

CONTRALORIA GENERAL DE LA REPUBLICA Y UNIVERSIDAD NACIONAL. EI Fondo Nacional del Café, Bogotá, 1988.

Economía Colombiana No. 191-193 de abril de 1987 y 221 de septiembre de 1989.

Estrategia Económica y Financiera No. 114. Septiembre de 1987.

Revista Negocios No. 69. Agosto de 1989.

Revista Nueva Frontera No. 740. Junio de 1989.

Revista Nueva Frontera No. 793. Julio 30 de 1990.

Revista Semana No. 372. Junio de 1989.

Revista Javeriana No. 548. Septiembre de 1988.

Enciclopedia Nueva Historia de Colombia. Editorial Planeta. Bogotá, 1989.

Publicaciones de la Federación Nacional de Cafeteros.

\section{Deuda Externa y Comercio Exterior.}

AUTORES VARIOS: Los Traficantes de la Pobreza. El F.M.I. y América Latina. El Ancora Editores. Bogotá, 1984.

SILVA Colmenares, Julio. Deuda Externa. Crisis y Confrontación. Ediciones Alborada. Bogotá, 1988.

OCAMPO, José Antonio y LORA, Eduardo. Colombia y la Deuda Externa. Tercer Mundo Editores. Bogotá, 1988. 
GARAY S., Luis Jorge. La Iniciativa Brady en la Evolución de la crisis de la deuda. Fescol. Bogotá, 1989.

RODDICK, Jacqueline. El Negocio de la Deuda Externa. El Ancora Editores. Bogotá, 1990.

\section{ARTICULOS:}

LOS BANCOS COMERCIALES Y EL DESARROLLO DE LA PERIFERIA: CONGRUENCIA Y CONFLICTO. Robert Devlin. Revista de La Cepal No. 9. Santiago de Chile, 1979.

RENEGOCIACION DE LA DEUDA LATINOAMERICANA: UN ANALISIS DEL PODER MONOPOLITICO DE LA BANCA. Robert Devlin. Revista de La Cepal No. 20. Santiago de Chile, 1983.

EL ESCENARIO INTERNACIONAL Y LA DEUDA EXTERNA DE AMERICA LATINA. Luciano Tomassini. Revista de La Cepal No. 24. Santiago de Chile, diciembre 1984.

POLITICA EXTERIOR Y NEGOCIACION FINANCIERA INTERNACIONAL: LA DEUDA EXTERNA Y EL CONSENSO DE CARTAGENA. Jorge E. Navarrete. Revista de La Cepal No. 27. Santiago de Chile, diciembre 1985.

EL PAPEL DE LAS ORGANIZACIONES MULTILATERALES EN LA RENEGOCIACION DE LA DEUDA. Hugo E. Souza, Revista Integración Latinoamericana. Enero-febrero 1986.

EL IMPACTO DE LA CONVERSION DE DEUDA EN CAPITAL. Michael Blackwell y Simón Nocera. Revista Finanzas y Desarrollo. Junio 1986.

LA DEUDA EXTERNA ÚN MENU DE OPCIONES PARA LOS PAISES DEUDORES. Robert Devlin. Revista Economía Colombiana No. 211-212. Noviembre-diciembre 1988.

DEUDA EXTERNA EN AMERICA LATINA Y EL CARIBE. Sela. Revista Economía Colombiana No. 211-212. Noviembre-diciembre 1988.

LA EVOLUCION DEL PROBLEMA DE LA DEUDA EXTERNA EN AMERICA LATINA Y EL CARIBE. Estudios e Informes de la Cepal. Santiago de Chile 1988.

DISYUNTIVAS FRENTE A LA DEUDA EXTERNA. Robert Devlin. Revista de La Cepal No. 37. Santiago de Chile. Abril, 1989.

\section{Reestructuración económica y ventaja comparativa dinámica}

BALCAZAR, A.V. Tecnología y crisis de la agricultura en Colombia. Economía Colombiana. 72-81.

BHAGWATI, J. Some recent trends in the pure theory of international trade. In International Trade Theory in a Developing World by Harrod R. \& D. Hague (1964), 1-30.

CALVO, H.S. \& M. MARTINEZ. La ventaja comparativa de la industria manufacturera colombiana. Bogotá, Proexpo, 1973, $40 \mathrm{p}$.

FRANSMAN, M. \& K. KING. Technological capability in the Third World. Macmillan, 1984, 404 p.

GUPTAL, L.C. Growth theory and strategy: new direction. Delhi, Oxford University Press, 1983, 162 p.

JOHNS, R.A. International trade theories and the evolving international economy. London, Frances Pinter, $1985,321 \mathrm{p}$.

JOHNSON, H.G. Effects of changes in comparative costs as influenced by technical change. In International Trade Theory in a Developing World by HARROD, R, \& D. HAGUE (1964), 96-112.

Factor endowments, international trade, and factor prices. In Readings in International Economics by American Economic Association (1968), 78-89.

KRUGMAN, P.R. (ed). Strategic trade policy and the New International economics. Cambridge, Mass., The MIT Press, 1987, 313 p.

LORA, E.T. Revisión de las teorías mercantilistas y clásicas sobre el comercio internacional. Cali, CIDSE, Monografia No. 1,60 p. 
MEIER, G.M. International trade and development. New York, Harper \& Row, 1963, 208 p. Cap. 2 Comparative Costs, 9-39.

NELSON, R.R. \& S.G. WINTER. An evolutionary theory of economic change. Harvard University Press, $1982,437 \mathrm{p}$.

PERRY, G.E. Políticas selectivas de promoción a las exportaciones. Coyuntura Económica, Vol. 8, No. 4, dic. 1978, 143-166.

ROBINSON, R. Factor proportions and comparative advantage. In Readings in International economics by American Economic Association (1968), 3-23.

ROJAS, G.L. Tipo de cambio y exportaciones agropecuarias. Boletín Socioeconómico (Cali, CIDSE, marzo 1987), No. 17, 51-71.

RYBCZYNSKI, T.M. Factor endowment and relative commodity price. In Readings in international economics by American Economic Association (1968), 72-77.

SAMUELSON, P.A. International factor price equalization once again. Ibidem, 58-71.

THOUMI, F. Estrategias de industrialización, empleo y distribución de empleo en Colombia. Coyuntura Económica, Vol. 9 No. 1, abril 1979.

VASQUEZ, E. Comentarios en torno al uso de la "Tecnologia" en las teorías económicas. Boletín Socioeconómico (Cali, CIDSE, marzo 1987), No. 17, 7-32.

\section{Condiciones para la participación humana en la Empresa}

TAYLOR, Frederick. Principios de Administración Científica.

MAYO, Elton. The social problem of an Industrial Civilization. Boston, Harvard, University Press, 1933.

MC GREGOR D. El aspecto humano de la Empresa. Nueva York, McGraw-Hill, 1960.

MONROY. Leonal. Las Etapas del Desarrollo del Ser Humano y su relación con el desarrollo del personal en las Organizaciones. Revista "Cuadernos de Administración". Universidad del Valle. Año 4, Noviembre 12, 1985.

SEVILLA. Andrés. Conferencias Mimeografiadas. Carvajal S.A. 